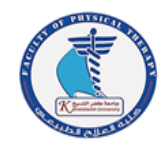

\title{
Different Transcutaneous Electrical Nerve Stimulation Modes in Relieving Chronic Pudendal Neuralgia in Females
}

\author{
Hala M. Hanafy ${ }^{1}$, Afaf M. Botla ${ }^{1}$, Mohamed F. Abo-El-Enan ${ }^{2}$, Mohamed Z. Mowafy ${ }^{*}$ \\ ${ }^{1}$ Department of Woman's Health, Faculty of physical therapy, Cairo University, Egypt. \\ ${ }^{2}$ Departement of obstetrics and gynecology, OM-El-Misrieen Hospital, Egypt.
}

*Correspondence to
Mohamed Z. Mowafy
Department of Woman's
Health, Faculty of physical
therapy, Cairo University,
Egypt.
Tel: 01155148076
Email:
m.z.mowafy123@gmail.com

Published online:

10-03-2020

\begin{abstract}
:
Purpose: to evaluate and compare the effects of two different transcutaneous electrical nerve stimulation (TENS) modes (conventional and SLR acupuncturelike TENS) on the female pudendal neuralgia.

Methods: Thirty female patients suffering from chronic pudendal neuralgia from out-clinic of the gynecological and obstetrical department, OM-EL MISRIEEN hospital, were participated in this study. Their ages ranged from 30 to 40 years old. They were assigned randomly into two equal groups; group (A) received the conventional TENS, group (B) received the SLR Acupuncture-likeTENS, in addition to the traditional physical therapy and medical care for both groups, duration of treatment was 20 minutes/session, every other day for 3 months. Measurement of the serum cortisol level (SCL) and the visual analogue scale (VAS) were recorded before and at the end of intervention.

Results: Results showed that both TENS modes were effective in improving the pudendal neuralgia as manifested by significant decreased SCL and VAS $(\mathrm{P}<0.05)$ at pre and post treatment program.

Conclusion: SLR acupuncture-like TENS more effective than the conventional TENS in improving the female pudendal neuralgia.

Key words: TENS; Pudendal neuralgia; Serum cortisol level; VAS.
\end{abstract}

\section{Introduction}

Pudendal neuralgia is an illness which involves neuropathy of the pudendal nerve. Pudendal neuralgia is caused by inflammation, compression and traction of the pudendal nerve. It may be associated with childbirth, pelvic surgery, strenuous exercise, abnormalities of the sacroiliac joint, and also, it is connected to age-related changes. The clinical features of this disease include hyperalgesia in the perineal region during sitting, which becomes worse during the day and could be relieved by standing or lying position, and the disease is associated with sexual dysfunction and difficulties in urination and/or defecation. The feeling of hyperalgesia can be felt in the vagina or rectum with elevated fever, swelling and/or foreign body sensation. Pudendal neuralgia is an intractable pain related to the pudendal nerve, which severely affects the quality of life and is hard to treat, (1).

Pudendal neuralgia (PN) due to pudendal nerve entrapment (PNE) is a well-known disease in medical community but both diagnosis and treatment may be delayed. The management of this harmful chronic pain remains a challenging task. Pudendal neuralgia is an increasing multifactorial condition, with a heavy impact on patient's quality of life. It consists of chronic perineal pain along the course of pudendal nerve, variably described by the patient, as mono, or bilateral, sometimes radiating to gluteus, genitals, or thighs. Women are more frequently affected than men. Recognized causes of pudendal neuralgia are floor muscle spasm, entrapment from sacrospinous, or sacrotuberous ligaments, pelvic trauma, or pelvic surgery (mesh, suture, or staples directly injuring the nerve). Pain limited to the 
territory of innervation of pudendal nerve, pain predominant during sitting, pain does not awaken patient from sleep, no objective sensory defects, positive effect of anesthetic infiltration of the pudendal nerve. Sensation of foreign rectal (or vaginal) body and worsening of pain during defecation, pain predominantly unilateral, and worsening throughout the day are complementary signs' (2).

The pudendal nerve arises from the ventral primary rami of S2, S3, and S4 of the sacral plexus. The pudendal nerve exits the pelvic cavity under the piriformis muscle through the greater sciatic foramen and descends ventral to the sacrotuberous ligament. The nerve then passes under the sacrospinous ligament medial to the ischial spine and re-enters the pelvic cavity through the lesser sciatic foramen. While under the levator ani muscle, the pudendal nerve courses ventrally through the pudendal canal of Alcock, a thickening of the obturator internus fascia. Within the ischioanal fossa, the pudendal nerve gives off two branches: the inferior rectal branch and perineal branch. The dorsal sensory nerve of the penis or clitoris forms the terminal branch. The inferior rectal branch of the pudendal nerve passes medially through the ischioanal fossa, providing motor supply to the external anal sphincter and sensory supply to perianal skin, (3).

Since the discovery of electricity, and before, current has been applied to the human flesh by a variety of methods to cure a multitude of afflictions. Electrical discharges from the black torpedo fish (Electric eels) were known to the Ancient Egyptians as well as to Hippocrates, for the treatment of headache and gout. The word electric was first used by William Gilbert (1544-1603), who was the first to classify and generalize the phenomenon of electricity in his book De Magnete. Kratzenstein (1746) wrote the first report on the use of electricity in medical therapy, so William Gilbert considered as the electricity father, while Kratzenstein considered as the electrotherapy father, $(4,5)$.

Transcutaneous electrical nerve stimulation (TENS) was introduced as a test for the gate-control theory of pain by (Melzack and wall, 1965). Wall went to recruit Sweet, who was head of neurosurgery at Harvard medical school to propose an experiment about the temporary abolition of pain, first on themselves and then, if that worked, on patients, Wall and Sweet published their work in 1967 (Wall and Sweet, 1967). TENS has rapidly been accepted as a standard modality in the treatment of pain and was introduced to the profession in the early $1970^{\text {th }}$. Practitioners, who have utilized TENS, properly have reported excellent results in many aspects of practice as pre and postoperative pain, non-united fracture pain and healing, obstetrics, dental and tempromandibular joint pain and other aspects $(6,7)$. The aim of our study is to compare between the effects of two different transcutaneous electrical nerve stimulation (TENS) modes (conventional and SLR acupuncture-like TENS) on the female pudendal neuralgia.

\section{Patients and Methods}

\subsection{Participants}

Thirty female patients suffering from chronic pudendal neuralgia from out-clinic of the gynecological and obstetrical department, OM-EL MISRIEEN hospital, were participated in this study. Their ages ranged from 30 to 40 years old. Those patients had chronic pudendal neuralgia that may be associated with childbirth, pelvic surgery, strenuous exercise, abnormalities of the sacroiliac joint, and may be connected to age-related changes that confirmed by physical exam, reproduction of pain with digital rectal examination, pressure at the level of the left ischial spine and positional nature of the pain and relief when sitting on a lavatory seat. Also, diagnosis was validated via the nerve conduction studies, using an intrarectal technique to measure the distal motor latency that objectively confirmed the diagnosis. They were assigned randomly into two groups; the first study group (A) was composed of 15 patients who received the conventional TENS in addition to the traditional physical therapy and medical care for 3 months. The second study group (B), that was composed of 15 patients who received the SLR (Acupuncture-like) TENS in addition to the traditional physical therapy and medical care for 3 months, $(8,9)$.

\subsection{Instrumentation:}

\subsubsection{Therapeutic equipment}

The treatment protocol was achieved by using a dual channel TENS stimulator: Model DH-808, made by DAE Han in Korea was utilized. Component of the TENS stimulator: Body case: 58 (width)-Dimension: 170 (height) 26 thickness in mm. Weight: 170 grams. Electrical rating: Power voltage: 9 volts direct current supplied by a DC adaptor (new general made in USA). Power consumed: $600 \mathrm{mw}$ (maximum). Frequency: $1 \mathrm{~Hz}-100 \mathrm{~Hz}$ Amplitude: $10 \mathrm{~mA}-100$ mA. Pulse width: $50-400$ sec. Wave form: Asymmetrical biphasic square pulse. Accessories: 4 body electrodes with 2 plugs and 2 adhesive belts. Self-adhesive electrodes: Dura-Stick II self-adhesive round electrodes (for use with most muscle stimulator, clinical electrotherapy, and transcutaneous electrical nerve stimulator) were used. Each electrode was half an inch (about 1.25 $\mathrm{cm})$ in diameter. Modulation: Intermittent changes in the TENS parameters of stimulation (frequency and intensity) were programmed by the manufacturer to vary about 10 percent periodically to overcome the 
accommodation process. in addition to the traditional physical therapy and medical care for 3 months (TENS application for 20minutes, three times weekly for 3 months), $(10,11)$

\subsubsection{Measurement equipment and tools}

2.2.2.1. Elexcess twenty ten device:

For blood serum analysis that was used for the measurement of serum cortisol level (Manufactured by Roch Company-Germany) $(12,13)$.

2.2.2.2. Visual Analogue Scale (VAS):

Pain level was assessed by visual analogue scale. VAS is a ten $\mathrm{cm}$ line anchored at each end with wards such as no pain and the worst pain possible. Both SCL and VAS were done before starting treatment (first record) and at the end of the total period of treatment after 3 months (second record), $(14,13)$.

2.3. Procedures of the study:

The experimental protocol was explained in detail for every patient before starting the initial assessment, and a written consent form was signed by each patient before starting. The treated patients were instructed to report any side effects during the treatment sessions.

2.3.1. Therapeutic Procedures: All patients in the 2 groups (A) and (B) received the same traditional physical therapy in the form of pelvic floor exercises and the same medical care.

Therapeutic Procedures for Group A and B (Conventional or SLR acupuncture- TENS application):

TENS treatment protocol: Position of subject and TENS electrodes placement:

The TENS was applied once day after day, three times per week for 3 months as a total period of treatment. Each treatment session was conducted for 20 minutes, two electrodes were positioned over the perineal area on the Centrum tendineum, while the other two electrodes were applied under the buttocks medially at the level of the ischial spines (medial to the ischial spines bilaterally) with the patient in comfortable supine hook-lying position with abducted hips, the TENS electrode surface area must be equal to half an inch (about $1.25 \mathrm{~cm}$ ) to minimize heat produced beneath electrodes to prevent skin burns. Also the interelectrode distance must not be less than the cross-sectional diameter of the electrode, to minimize current density between electrodes, so heat produced either beneath or between must not exceed the safe limits to avoid skin burn, the 4 electrodes were of the adhesive type that used over the recommended areas $(15,11)$.

Stimulation parameters of the conventional TENS mode application in the first study group (A) were Square wave form, frequency from $80-100 \mathrm{~Hz}$ and an intensity from $10-30 \mathrm{~mA}$, associated with a pulse width from 50-60 $\mu \mathrm{sec}$, producing a comfortable perceptible paresthesia (tingling) without significant muscle contractions or fasciculations $(6,15)$.

Stimulation parameters of the SLR (Acupuncturelike) TENS mode application in the second study group (B): were square wave form, frequency of less than $10 \mathrm{~Hz}$ and an intensity from 30 to $80 \mathrm{~mA}$, associated with a pulse width from 300-400 $\mu \mathrm{sec}$, producing a rhythmic and visible muscle contractions, that were strong to the level of tolerance in the same position of subject and TENS electrodes placement (15 minutes for three times weekly for 3 months) $(4,5)$

\subsubsection{Measurement tools:}

\subsubsection{Serum cortisol level measurement: (SCL):}

Normal cortisol level ranged from $9-25 \mu \mathrm{g} / \mathrm{dL}$ at morning and patients with painful conditions tended to have higher than normal SCL, estimation of serum cortisol level was carried out before and after 3 months of treatment program. A venous blood sample of $8 \mathrm{CC}$ was taken at the morning, centrifuged and stored at $20^{\circ} \mathrm{C}$ till analyzed $(4,9,22)$

2.3.2.2. Visual analogue scale (VAS): was done before and after the treatment program $(8,13)$.

\section{Data analysis}

Serum cortisol level and visual analogue scale records were measured before treatment and after cessation of the treatment program in both groups after 3 months of treatment. Collected data were fed into computer for the statistical analysis; descriptive statistics as mean, standard deviation, minimum and maximum were calculated for each group. The t-test was done to compare the mean difference of the two groups before and after application and within each group. Alpha point of 0.05 was used as a level of significance' $(16,17)$.

\section{Results}

In the present study, effects of two different transcutaneous electrical nerve stimulation modes (conventional TENS and SLR acupuncture-like TENS) on the female pudendal neuralgia were investigated. As shown in table (1) and figure (1), the mean value of the serum cortisol level in $\mu \mathrm{g} / \mathrm{dl}$ (SCL) before treatment was $(36.440 \pm 0.404) \mu \mathrm{g} / \mathrm{dl}$ in the first study group (Conventional TENS), while after treatment was $(36.106 \pm 0.399) \mu \mathrm{g} / \mathrm{dl}$. These results revealed only significant reduction in SCL, $(\mathrm{P}<0.05)$. But in the second study group (SLR acupuncture-like TENS), the mean value of the serum cortisol level in $\mu \mathrm{g} / \mathrm{dl}$ (SCL), before treatment was $(36.1622 \pm 0.5525) \mu \mathrm{g} / \mathrm{dl}$, while after treatment was $(26.6144 \pm 0.5445) \mu \mathrm{g} / \mathrm{dl}$, and these revealed a highly significant decrease in SCL, $(\mathrm{P}<$ $0.0001)$. 
Table (1): Comparison of the mean values of the SCL in $\mu \mathrm{g} / \mathrm{dl}$, before and after treatment in both groups

\begin{tabular}{|c|c|c|c|c|c|c|c|}
\hline & \multicolumn{2}{|c|}{ Before treatment } & \multicolumn{2}{|c|}{ After treatment } & \multirow[b]{2}{*}{$\begin{array}{c}\text { Mean } \\
\text { difference }\end{array}$} & \multirow[b]{2}{*}{ T.value } & \multirow[b]{2}{*}{ P.value } \\
\hline & $\begin{array}{c}\text { Mean } \\
(\mu \mathrm{g} / \mathrm{dl})\end{array}$ & $\pm \mathrm{SD}$ & $\begin{array}{l}\text { Mean } \\
(\mu \mathrm{g} / \mathrm{dl})\end{array}$ & $\pm \mathrm{SD}$ & & & \\
\hline $\begin{array}{c}\text { Group } \\
\text { A }\end{array}$ & 36.44 & 0.40 & 36.10 & 0.39 & -0.33 & -2.28 & $\begin{array}{c}<0.03 \\
(\mathrm{~S})\end{array}$ \\
\hline $\begin{array}{c}\text { Group } \\
\text { B }\end{array}$ & 36.16 & 0.55 & 26.61 & 0.54 & -9.54 & -47.67 & $\begin{array}{l}0.00 \\
\text { (HS) }\end{array}$ \\
\hline
\end{tabular}
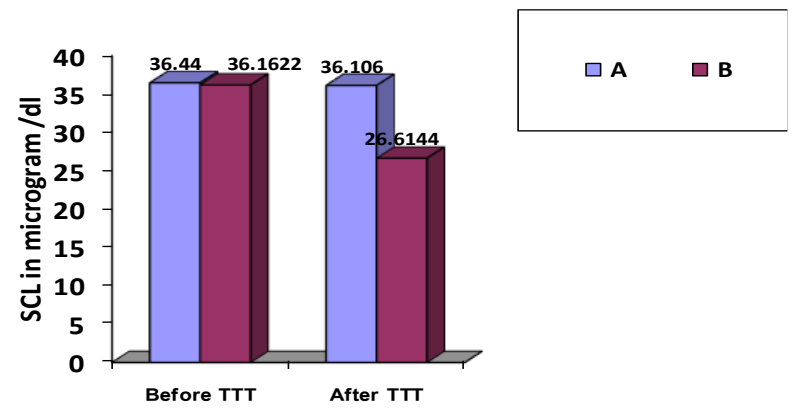

Fig (1) : Mean values of SCL in $\mu \mathrm{g} / \mathrm{dl}$ before and after treatment in both groups.

In the present study, effects of two different transcutaneous electrical nerve stimulation modes (conventional TENS and SLR acupuncture-like TENS) on the female pudendal neuralgia were investigated. As shown in table (2) and figure (2), the mean value of the visual analogue scale in degrees (VAS) before treatment was $(8.700 \pm 0.155)$ degrees in the first study group, while after treatment was $(8.524 \pm 0.242)$ degrees. These results revealed only significant reduction in VAS, $(\mathrm{P}<0.05)$. But in the second study group, the mean value of the visual analogue scale in degrees (VAS), before treatment was $(8.720 \pm 0.132)$ degrees, while after treatment was $(3.500 \pm 0.118)$ degrees, and these revealed a highly significant decrease in VAS, $(\mathrm{P}<0.0001)$.

Table (2): Comparison of the mean values of the VAS in degrees, before and after treatment in both groups

\begin{tabular}{|c|c|c|c|c|c|c|c|c|}
\hline & \multicolumn{3}{|c|}{ Before treatment } & \multicolumn{2}{|c|}{ After treatment } & \multirow[b]{2}{*}{$\begin{array}{c}\text { Mean } \\
\text { differe } \\
\text { nce }\end{array}$} & \multirow[b]{2}{*}{$\begin{array}{l}\text { T.val } \\
\text { ue }\end{array}$} & \multirow[b]{2}{*}{$\begin{array}{c}\text { P.valu } \\
\mathrm{e}\end{array}$} \\
\hline & $\begin{array}{r}\mathrm{Mea} \\
\text { (degre }\end{array}$ & & $\begin{array}{c} \pm \\
\mathrm{SD}\end{array}$ & $\begin{array}{c}\text { Mean } \\
\text { (degrees) }\end{array}$ & $\pm \mathrm{SD}$ & & & \\
\hline $\begin{array}{l}\text { Group } \\
\text { A }\end{array}$ & 8.7000 & & 0.15 & 8.52 & 0.24 & 0.17 & 2.37 & $\begin{array}{c}<0.02 \\
(\mathrm{~S})\end{array}$ \\
\hline $\begin{array}{l}\text { Group } \\
\text { B }\end{array}$ & 8.720 & 0.13 & & 3.50 & 0.118 & 5.20 & 113.7 & $\begin{array}{l}0.00 \\
\text { (HS) }\end{array}$ \\
\hline
\end{tabular}

\section{Discussion}

Since the discovery of electricity and before, current has been applied to the human flesh by a variety of methods to cure a multitude of afflictions. Electric eels (Black torpedo fish) were known to the Ancient Egyptians as well as to Hippocrates, for the treatment of headache and gout. The word electric was first used by William Gilbert (1544-1603), who was the first to classify and generalize the phenomenon of electricity in his book De Magnete. Static discharges were also well known after the invention of the Leyden jar in 1745. In the same year Kratzenstein first induced muscle contraction by static electricity. The next year he wrote the first report on the use of electricity in medical therapy, so William Gilbert considered as the electricity father while Kratzenstein, considered as the electrotherapy father, $(15,4)$.

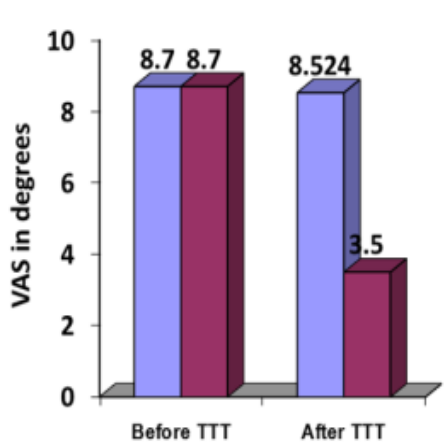

$\square \mathrm{A}$

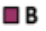

Fig (2) : Mean values of VAS in degrees before and after treatment in both groups.

The use of electricity as a form of therapy is a wellestablished procedure in medicine but also has as part of its history some crude forms of application. as long ago as $400 \mathrm{BC}$, the black torpedo fish or the electric eel, was used to impart electric shocks to persons standing in water to treat gout, headaches, asthma, hemorrhoids, and many other medical maladies. Later, names like Kratzenstein, Volta, Galvani, Faraday, Lovett, Wesley, Birch, Melzack, Wall, Sweet, Shealy, Long and Kotz became important through their development of concepts and devices that generated and / or measured "electric shocks" for the therapeutic purposes. The electric potential, or electric current, generated by the TENS unit must be transmitted to the patient before it can cause nervous system modulation secondary to its effects. At this time, the current must be transmitted from the TENS generator by way of lead wires interposed between the TENS generator and electrodes on the skin. TENS may be used successfully when electrodes are placed over the site of pain, trigger, acupuncture or motor points, over the course of the peripheral nerve or paravertebrally' $(5,10)$. 
TENS is an effective, noninvasive, nonaversive, nonaddictive method of managing pain, muscle guarding and dysfunction of the pain cycle as well as the internal changes that accompanied the pain cycle can be managed or at least reduced by TENS application. As pain produces a state of muscle tension that results in a diminished blood supply within the painful area (or a state of ischaemia), increased metabolites, decreased oxygen supply, decreased lymphatic clearing, decreased nutrient supply, increased muscle fatigue, inflammation and oedema. All these internal changes can lead to the progressive amplification of the pain cycle which can be prevented or reduced by TENS' $(15,4)$.

Although idiopathic urogenital and anorectal pain syndromes are not uncommon, effective treatments remain elusive for this patient group. Pain in these areas of the body can be embarrassing for the patient, limiting the desire to discuss the symptoms with the physician; physicians also may not be familiar enough with these pain syndromes, leading to misdiagnosis. Poorly understood pain syndromes, including idiopathic vulvodynia, prostatodynia, orchialgia, idiopathic proctalgia, coccydynia, levator ani syndrome, and urethral syndrome, share many common features, including pain in the distribution of one or both pudendal nerves. A case report is presented to suggest that pudendal nerve entrapment (PNE) can be a cause of chronic perineal pain refractory to conventional treatments $(18,19)$.

In neuralgia, pain can occasionally be reproduced by gently tapping over an area of point tenderness (Tinel sign) or by extending the thigh or hip. If a local entrapment or neuroma is responsible for the pain, a local block of the pudendal nerve alleviates the symptoms. If the pain is not relieved, the S-2, S-3 and S4 nerve plexus can be blocked through a paravertebral route with $0.5 \%$ bupivacaine and $0.75 \%$ lidocaine with epinephrine 1:200,000.by performing separate blocks and observing for pain relief. PNE can cause pain in the rectum, scrotum (labia), or penis (clitoris). At onset, pain is exacerbated in the seated position, except on a lavatory seat, and relieved by standing or lying down. Diagnostic techniques, including CT-guided nerve blocks and nerve conduction studies, can confirm the diagnosis. Once diagnosed, guided corticosteroid injections and surgical intervention can provide relief and even cures for this formerly refractive population. At present, it is unknown what fraction of cases of idiopathic urogenital and anorectal pain syndromes are related to PNE. However, because accurate diagnostic methods and effective treatment options for PNE do exist, these diagnostic methods may be appropriate before concluding a patient has an idiopathic perineal pain syndrome, $(20,21)$.
Pudendal Neuralgia is a painful neuropathic condition that is caused by inflammation of the pudendal nerve. Triggers for the sensitivity include trauma secondary to childbirth, surgery, cycling, squatting exercises, bio-mechanical abnormalities (e.g., sacroiliac joint dysfunction, pelvic floor dysfunction), chronic constipation, repetitive vaginal infections and direct falls on the tailbone. Pudendal neuralgia is known in some circles as (cyclist's syndrome), (pudendal canal syndrome) or (Alcock's syndrome). Primary symptoms of Pudendal Neuralgia include pelvic pain with sitting that may be less intense in the morning and increase throughout the day. Symptoms may decrease when standing or lying down. The pain can be perineal, rectal or in the clitoral / penile area; it can be unilateral or bilateral, sexual dysfunction in women manifested as pain or decreased sensation in the genitals, perineum or rectum. Pain may occur with or without touch. It may be difficult or impossible for the woman to achieve orgasm, (22).

Results of the present study showed that was a highly significant decrease between the means of the second record SCL (2) (after three months of the conventional TENS application) and the first record SCL (1) (pre- application of the conventional TENS) $(\mathrm{P}<0.0001)$.

Findings of the present study showed that there was a highly significant decrease between the means of the second record of SCL (2) (after three months application of the SLR acupuncture-like TENS) and the first record of SCL (1) (pre-application of the SLR acupuncture-like TENS), in the second study group (SLR acupuncture-like TENS application group) ( $\mathrm{P}>0.0001)$.

Comparison between the means of the first pretreatment records of the SCL in the two groups revealed that there were non-significant differences in the first pre-treatment records of SCL, between the first and the second study groups $(\mathrm{P}>0.05)$.

But comparison between the means of the second records of the SCL in the two groups showed that there was highly significant decrease in the second records of SCL, between the second study and the first study groups $(\mathrm{P}<0.0001)$. in favoring to the second study group of the SLR acupuncture-like TENS application.

Findings of the present study showed that there was a highly significant decrease between the means of the second record VAS (2) (after three months of the conventional TENS application) and the first record VAS (1) (pre- application of the conventional TENS) $(\mathrm{P}<0.0001)$.

Findings of the present study showed that there was there was a highly significant decrease in VAS between means of the VAS (1) and VAS (2), in the second study group (SLR acupuncture-like TENS application) $(\mathrm{P}>0.0001)$. 
Comparison between means of the first pre-treatment records of VAS in the 2study groups revealed that there was non-significant difference in the first pretreatment records of VAS, between the first and second study groups $(\mathrm{P}>0.05)$.

But comparison between the means of the second records of VAS in the two groups showed that there was a highly significant decrease in the mean values of the VAS second records between the second and first study groups $(\mathrm{P}<0.0001)$.

Both the conventional TENS and the SLR, acupuncture-like TENS produce sympathetic inhibition resulting in improving the peripheral circulation via lowering the vascular resistance and increasing the skin, muscle and nerve circulation. The vasodilating effect of the SLR, acupuncture-like TENS is more stronger than that of the conventional TENS, as the SLR, acupuncture-like TENS produce prolonged and wide spread sympathetic inhibition resulting in improved skin micro-circulation as well as the muscle and nerve circulation with increased skin temperature, accelerate healing, maturation of the burn wounds and improving the nerve function, while the conventional TENS effect is not prolonged and relatively of short aftereffect. The SLR, acupuncture-like TENS increase blood supply via vasodilatation. The peripheral vasodilatation promotes tissue perfusion and elevates skin, muscle and nerve temperature. The SLR, acupuncture-like TENS increase the peripheral vasodilatation dramatically in cold extremities with a 7 to $10{ }^{\circ} \mathrm{C}$ increase in skin temperature, that persisted for at least 4 to 8 hours after TENS application, $(7,15)$.

The SLR, acupuncture-like TENS decrease the arterial levels of catecholamines. Catecholamines reduction resulted in decrease of both sympathetic tone and the hypermetabolic response of burned patients leading to the reduction of the vascular resistance, improving skin, muscle and nerve circulation as well as the nerve functions' $(10,14,16)$. While the SLR, acupuncture-like TENS decrease pain via the release of the endogenous beta endorphin. SLR, acupuncture-like TENS stimulate the small (pain) afferent and (motor) efferent fibers and has a relatively long aftereffect, as well as increase the beta endorphin level within the cerebrospinal fluid, while the conventional TENS stimulate the large (proprioceptive) afferent fibers and has a relatively short after-effect. The vasodilating effect of both conventional and SLR, acupuncture-like TENS may represent another mechanism by which TENS provide pain relief, $(4,5)$. There is a linear correlation between the peripheral vasodilatation and the skin temperature, as the increase in peripheral vasodilatation is associated with an elevation of the skin temperature and vice versa, also there is a linear correlation between the skin temperature, subcutaneous, intramuscular and intraneural temperatures as well as the nerve conduction velocity is related to the skin, subcutaneous, intramuscular and intraneural temperatures, so the nerve conduction velocity is related to the peripheral vasodilatation degree. as the nerve conduction velocity increases almost linearly, by $2.4 \mathrm{~m} / \mathrm{s}$ or approximately $5 \%$ for every degree centigrade of temperature increase' (6).

The results of this study supports the expectation that both the SLR, acupuncture-like TENS (to greater extent) and the conventional TENS (to lesser extent), are significantly effective in decreasing sympathetic tone, decreasing vascular resistance, improving the skin, subcutaneous, intramuscular and intraneural temperatures via the prolonged and widespread vasodilatation that improving the intraneural blood supply, improving pudendal neuralgia as evidenced by the highly significant decreases in the SCL and VAS.

These significant differences, between the second study group (SLR acupuncture-like TENS application) and the first study group (Conventional TENS application), which were in the form of highly significant decreases in the SCL and VAS were consistent with those observed and recorded by Shimazu et al(10).

Eventually, after the discussion of the results and according to reports of the previous investigators in fields related to this study, it can be claimed that the application of both the SLR, acupuncture-like TENS (to greater extent) and the conventional TENS (to lesser extent), are significantly effective and had a valuable effects on the pudendal neuralgia as evidenced by the highly significant decreases in SCL and VAS. But the SLR acupuncture-like TENS was more effective than the conventional TENS.

\section{Conclusion}

Application of both modes of TENS had a valuable effect on the pudendal neuralgia as evidenced by the highly significant decreases in SCL and VASA. But the SLR acupuncture-like TENS was more fruitful and effective than the conventional TENS.

Funding: Not funded.

Conflict of Interests

Authors declare no potential conflicts of interest.

Acknowledgments

We would like to thank all individuals who contributed to the completion of this work, especially participants.

\section{References}

1. Amarenco GS, Ismael SS, Bayle BH. Electrophysiologic analysis of pudendal neuropathy following traction. Muscle Nerve. 2008;24:116-119. 
2. Robert RA, Bensignor MA, Labatt JJ. Chronic perineal pain and the Pudendal Nerve Entrapment. Ann Orthop Ouest. 2009; 31:71-74.

3. Ramsden CE, Harmon RL, Renney KM. Pudendal nerve entrapment as source of intractable perineal pain. Am J Phys Med Rehabil.2003; 82:479-484.

4. Roberts HJ. Transcutaneous electrical nerve stimulation in the management of pancreatitis pain. South Med J. 2007;71:369.

5. Schonberg Fl, Ager WA, Carter-Baker SA. Transcutaneous Electrical Nerve Stimulation for Postlaparotomy Pain. Phys Ther. 2003; 63(2): 188-193.

6. Akyuz GA, Guven ZM, Kayhan OI. The Effect of Conventional TENS on Somato Sensory Evoked Potentials. Electromy Clin Neurophyiol.2005; 35: 371-376.

7. McCallum MI, Phillips AM. Transcutaneous Electrical Nerve Stimulation in the Management of Acute Postoperative Pain. Br J Anaesth. 2004; 61: 308-312.

8. Hokinson EC. Measurement of pain. The Lancet.2004; 2(7889):1127-1131.

9. Ponders FF. Electro acupuncture analgesia could be mediated by at least two main relieving mechanisms endorphin and non-endorphin systems. Life. 2009;26:631.

10. Shimazu KH, Kawakawa KJ, Makita MK. Analgesic Effects Induced by TENS and Electro acupuncture with Different Types of Stimulating Electrodes on Deep Tissues in Human Subjects. Pain. 2005;63:181-187.

11. String JF. Transcutaneous Electrical Nerve Stimulation in chronic pancreatitis. Anaesthesia.2008; 23:219-223.

12. Tu FA. Physical therapy evaluation of patients with chronic pelvic pain: a controlled study. Am J Ostetb Gynecol. 2008; 198:272.e1-272.e7.

13. Woolf JK. Endogenous opioid peptides and pain mechanisms: A complex relationship. Nature.2009;11:122-129.

14. Rhame EE, Levey KA, Gharibo CG. Successful treatment of refractory pudendal neuralgia with pulsed radiofrequency . Pain Physician. 2009; 12 (3): 633-638.

15. Renner HF. Transcutaneous and peripheral nerve stimulation for chronic pain states.Surgical neurology.2009;2:99-106.

16. Hinton PR. Statistics Explained $2^{\text {nd }}$ Ed. Rutledge Taylor and Francis Group London. 2004; pp149155.

17. Pipkin FB. Medical statistics made easy. Edinburgh. London. Mel Bourne and New York. 1984.

18. Oberpenning HJ. The Alcock syndrome: Temporary penile insensitivity due to compression of the pudendal nerve within the Alcock canal. J Urol. 2008; 22:67-76.

19. Robert RA, Labat JJ, Martin SK. Costargent; Bases anatomiques de la chirurgie du nerf pudendal. Conséquences thérapeutiques dans certaines algies périnéales. Lyon Chir. 2005; 89:183-187.

20. Schaeffer GH. Biofeedback, pelvic floor reeducation and bladder training for male chronic pelvic pain syndrome. Department of Urology, North Western University Medical School, Chicago, Illinois. 2000;IL 60611, 11-715.

21. Shafik A. A new concept of the anatomy of the anal sphincter mechanism and the physiology of defecation. XXVIII - Complete rectal prolapse: a technique for repair. Coloproctology.1998; 9:345-352.

22. Tagnetti FD, Poppi ME, Gaist GT. Pudendal neuralgia due to solitary neurofibroma: Case report. J Neurosurg. 2004;56:732-3. 